\title{
CONFLICTOS ENTRE ESCOLARES DE EDUCACIÓN SECUNDARIA Y VISIONES DEL PROFESORADO. ${ }^{1}$
}

\author{
CRISTINA DEL BARRIO 2 \\ Universidad Autónoma de Madrid.
}

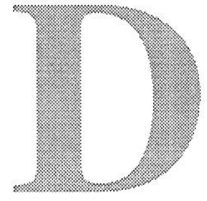

e modo similar a lo sucedido con otros aspectos relacionados con la educación, la toma de conciencia de la importancia de las relaciones interpersonales y el tipo de convivencia de los colegios e institutos ha surgido de los problemas y conflictos vividos en las últimas décadas, y no tanto del convencimiento acerca del papel fundamental de estos factores en el desarrollo y el aprendizaje de los estudiantes. Así pues, han sido los conflictos entre profesores y alumnos y entre los propios compañeros, sobre todo en determinados casos muy graves que han tenido gran eco en los medios de comunicación, los que han despertado la alarma en la comunidad escolar y en la sociedad en general, lo que confirma esa tendencia de la educación a reaccionar a los problemas más que a prevenirlos.

Desde la psicología del desarrollo se ha insistido en la importancia de las relaciones con los otros, en especial con los iguales, como factor de desarrollo social y moral de la persona, (para una revisión, véase Rubin, Bukowski y Parker 1998). Las relaciones, en su triple dimensión conductual, afectiva y cognitiva -es decir, las conductas convergentes o divergentes con las de otros, o su ausencia, así como las emociones ligadas a ellas y la reflexión que suscitan-contribuyen a la construcción de cada uno como ser humano. La escuela proporciona un contexto único de interacción y relación con compañeros y adultos. Y si hablar del constructivismo es un tópico, quizá no se ha debatido con la suficiente extensión cómo se aplica este enfoque al desarrollo social.

Por otro lado, el clima de relaciones interpersonales que se respira en un centro influye de forma determinante en la calidad de su funcionamiento en general y del aprendizaje de los alumnos. En particular la

1 Este texto es una versión resumida de otro incluido en el Informe Educativo 2001: La calidad del sistema educativo. Madrid. Santillana.

2 Cristina del Barrio es profesora del Depto. de Psicología Evolutiva y de la Educación de la Universidad Autónoma de Madrid. 
educación en valores, vinculada al desarrollo social, es claramente una de las áreas educativas determinada por el clima de convivencia de la institución escolar. Los valores no pueden enseñarse mediante procedimientos meramente declarativos. Contar en el aula lo que debe hacerse no basta para que los alumnos vayan adquiriendo conductas más adecuadas ni construyendo una capacidad de juicio moral que les permita regir su comportamiento de forma autónoma de acuerdo con un sistema axiológico propio. Como han señalado Martín y Mauri (2001), uno de los mecanismos de influencia educativa más relevantes de la institución escolar como tal consiste en que su organización y su cultura enseñan por sí mismas valores a los alumnos. Lo que se fomenta o se rechaza de forma explícita o implícita, las normas que rigen en la institución -tanto desde el punto de vista de su contenido como del procedimiento por el que lleguen a establecerse-, los modelos de comportamiento que predominan, los compromisos que presiden la práctica del centro..., en último término, el entramado de relaciones interpersonales que se producen y los valores que dejan traslucir, configuran la matriz a partir de la cual los alumnos irán construyendo su desarrollo social y moral.

Los problemas de convivencia, tanto los que se manifiestan en conductas agresivas como los comportamientos disruptivos, obligan a utilizar una gran parte de la energía y del tiempo escolar en intentar solucionarlos, consumiendo con ello el tiempo necesario para desarrollar el resto de los contenidos de aprendizaje. Aprender exige un clima de relación caracterizado por la serenidad, la confianza, la seguridad y la curiosidad, condiciones difícilmente compatibles con relaciones interpersonales deterioradas como consecuencia de conflictos.

Por ello, además de determinar los datos sobre el nivel de conflictos que tienen lugar en los centros de nuestro país, es preciso conocer las opiniones de alumnos y profesores sobre sus causas y la mejor forma de solucionarlos, datos que pueden ayudar a planificar políticas educativas de las administraciones y medidas gestionadas por los propios centros. Ambos objetivos estaban presentes en el estudio que se presenta a continuación.

\section{LOS CONFLICTOS EN LOS CENTROS DE EDUCACIÓN SECUNDARIA EN ESPAÑA.}

Los datos que aquí se presentan forman parte de un estudio más amplio realizado en el año 1999 por encargo del Defensor del Pueblo en colaboración con $\mathrm{UNICEF}^{3}$. Este informe se lleva a cabo a raíz de la petición de la Comisión Mixta Congreso-Senado del Parlamento es- 
pañol a la Oficina del Defensor de una información rigurosa acerca de la incidencia de conflictos violentos en los centros de secundaria en el conjunto del estado español.

\subsection{Características del estudio realizado.}

La muestra escolar del estudio estaba constituida por 3.000 estudiantes de educación secundaria, pertenecientes a 300 centros de secundaria elegidos de acuerdo a tres variables: contexto (urbano-rural), titularidad (pública-privada) y distribución proporcional en las diferentes comunidades autónomas. ${ }^{4}$ En cada una de las escuelas se seleccionaron al azar dos estudiantes - un chico y una chica- de cada uno de los cuatro cursos de la etapa de Educación Secundaria Obligatoria (ESO) o de los cursos correspondientes en el sistema de la Ley General de Educación. Se eligieron otros dos estudiantes más en cada escuela para completar el número necesario en la muestra, rotando el curso del que se tomaban.

Los diez alumnos y alumnas contestaban, en una sala de la escuela diferente a su aula, un cuestionario, elaborado para esta investigación tras el correspondiente estudio piloto. La contestación al cuestionario se llevaba a cabo en presencia de un encuestador que podía así resolver posibles dudas. Además de los datos de los alumnos, en la investigación se recogieron las opiniones de los 300 jefes de estudio de los centros de la muestra, que rellenaron también un cuestionario complementario al de los estudiantes. En este caso, los profesores lo rellenaban a solas y lo entregaban posteriormente al encuestador.

$\mathrm{El}$ estudio se centró fundamentalmente en un tipo de conflicto, el maltrato entre compañeros por abuso de poder (bullying), si bien también se realizaron, aunque con menor profundidad, preguntas acerca de otro tipo de problemas en las relaciones personales de los miembros de la comunidad escolar. De acuerdo con Olweus (1993), entenderíamos este tipo de comportamiento violento como una «conducta de persecución física o psicológica que realiza el alumno o alumna

Informe del Defensor del Pueblo sobre violencia escolar: el maltrato entre iguales en la Educación Secundaria Obligatoria. Elaborado por C. del Barrio, E. Martín, I. Montero, L. Hierro, I. Fernández, H. Gutiérrez y E. Ochaíta, por encargo de la Comisión Española de UNICEF. Ha sido publicado en Madrid, en Publicaciones de la Oficina del Defensor del Pueblo en el año 2000.

4 Las Comunidades Autónomas presentes en este estudio eran aquéllas que en el momento de llevar a cabo la investigación (curso 1998-1999) tenían ya competencias plenas en cducación: Andalucía, Baleares, Canarias, Cataluña, Navarra, País Vasco y Valencia. El resto de las Comunidades se analizaron conjuntamente al depender a efectos educativos todavía del Ministerio de Educación y Cultura. 
contra otro, al que elige como víctima de repetidos ataques. Esta acción, negativa e intencionada, sitúa a las víctimas en posiciones de las que dificilmente pueden salir por sus propios medios. La continuidad de estas relaciones provoca en las víctimas efectos claramente negativos: disminución de su autoestima, estados de ansiedad e incluso cuadros depresivos, lo que hace difícil su integración en el medio escolar y el desarrollo normal de los aprendizajes».

Esta definición hace hincapié en la intención del agresor, la reiteración de la conducta y el desequilibrio de poder entre la víctima y quien(es) agrede(n). Estando siempre presentes estas características, el maltrato entre iguales por abuso de poder toma, sin embargo, formas muy distintas que es preciso conocer para poder planificar la intervención educativa adecuadamente. Uno de los objetivos del estudio fue identificar la incidencia de las diferentes conductas que adoptaba el maltrato entre escolares de secundaria.

\subsection{La incidencia del maltrato.}

A partir de los datos ofrecidos por los alumnos, recogidos en la tabla I, puede afirmarse que todos los tipos de maltrato por los que se ha preguntado tienen lugar en los centros docentes de secundaria españoles, si bien con un nivel de incidencia muy distinto. La frecuencia decrece en la medida en la que aumenta la gravedad. ${ }^{5}$ Así, se produce un mayor número de abusos por agresión verbal y exclusión social, seguido de agresiones físicas indirectas (i.e. robar, esconder cosas, romperlas), conductas de amenaza para intimidar, agresiones físicas directas (i.e. pegar) y, en mucha menor medida, obligar a otro bajo amenazas a hacer cosas que no desea (o chantaje), acoso sexual y amenazas con armas (del Barrio et al., 2002).

La tabla 1 presenta la diferente incidencia de los tipos de maltrato desde las distintas perspectivas que adoptan los estudiantes que participaron en el estudio: agresores, víctimas y testigos; es decir, el porcentaje de estudiantes que reconocen haber inflingido, sufrido o presenciado maltrato. Hay un número superior de participantes en el estudio que declaran ser autores de agresiones verbales, exclusión social, y agresión física directa (i.e. pegar), comparado con el número de participantes que dicen sufrir esas agresiones. Sin embargo, en otros tipos de agresiones -las dirigidas a las propiedades de los compañeros (esconder, romper o robar cosas), las amenazas y el acoso sexual-

5 El concepto de gravedad debe ser empleado con mucha prudencia, ya que las secuelas psicosociales de algunas de las conductas de exclusión social o agresión verbal pueden ser también muy importantes. 
Tabla 1: Porcentaje de estudiantes de secundaria que afirman haber presenciado, sufrido o perpetrado diferentes modos de maltrato entre compañeros $(N=3000)$.

\begin{tabular}{|l|c|c|c|}
\hline Comportamiento & Testigo (\%) & Víctima (\%) & Agresor (\%) \\
\hline Ignorar & 79 & 14,9 & 38,7 \\
\hline Excluir & 66,5 & 10,7 & 13,7 \\
\hline Insultar & 91,6 & 38,5 & 45,5 \\
\hline Poner motes & 91,3 & 37,2 & 37,9 \\
\hline Hablar mal de otro/a & 88,3 & 34,9 & 38,5 \\
\hline Esconder cosas & 73,9 & 21,8 & 13,5 \\
\hline Romper cosas & 37,6 & 4,4 & 1,3 \\
\hline Robar cosas & 39,5 & 7,3 & 1,5 \\
\hline Pegar & 59,6 & 4,8 & 7,2 \\
\hline Amenazar para amedrentar & 66,2 & 9,7 & 7,4 \\
\hline Obligar a hacer cosas mediante amenazas & 12,6 & 0,8 & 0,4 \\
\hline Amenazar con armas & 6,2 & 0,7 & 0,4 \\
\hline Acosar sexualmente & 7,6 & 2 & 0,6 \\
\hline
\end{tabular}

son más los escolares que afirman sentirse agredidos que los que dicen ser autores de ellas.

Algunas de las manifestaciones de maltrato, entre ellas las consideradas más graves, por ejemplo, acosar sexualmente, amenazar con armas, se producen con una frecuencia muy baja. Este dato no impli$\mathrm{ca}$, sin embargo, que no deba darse importancia a estos hechos. Por el contrario, aunque los niveles de incidencia sean bajos, al tener una repercusión tan nociva, especialmente en las víctimas, pero también en quienes las llevan a cabo y en quienes las presencian, debe intervenirse para atajarlas y diseñar medidas de prevención para que no lleguen a producirse. Por otra parte, hay que prestar una atención especial a las agresiones consideradas "menos graves". Muchas de ellas, especialmente las que implican exclusión social, suponen importantes daños psicológicos para los escolares (van der Meulen et al., 2002).

Por otra parte, si comparamos la información ofrecida por el conjunto de estudiantes y lo que contestan los profesores acerca de la frecuencia con la que creen que se producen los diferentes tipos de maltrato, se encuentra una incidencia claramente superior en las respuestas de los docentes. En algunos tipos de maltrato, aunque no en todos ellos, se encuentran cifras más altas incluso que las de los alumnos cuando éstos informan como testigos. Parece lógico que el perfil de respuesta de los docentes se asemeje básicamente al de los testigos, ya que el papel habitual que desempeñan en este tipo de conflictos no es el de víctima ni el de agresor, sino más bien el de quien presencia la agresión. La alta incidencia hallada podría deberse al hecho de que 
los profesores tienen acceso a un mayor número de posibles situaciones de maltrato, sea en los distintos grupos en que imparten clase o en todo el centro.

\subsubsection{Diferencias en la incidencia.}

Con respecto a las variables que producen una incidencia diferente, tres son las que resultan influyentes, según los datos españoles: género, curso y titularidad del centro.

Así, confirmando los datos encontrados en otros muchos estudios, el maltrato es un fenómeno fundamentalmente masculino. En general, los chicos agreden y sufren mayor número de agresiones que las chicas, con una excepción: la conducta de hablar mal de otros se da más entre las chicas, y parece no haber diferencias entre uno y otro género a la hora de ignorar o ser ignorados por otros. Con respecto al curso, las agresiones se producen en primer curso en un número significativamente mayor y -de modo menos marcado- en segundo y en cuarto en una proporción significativamente menor. La diferencia entre titularidad pública y privada se manifiesta de un modo estadísticamente significativo en muy pocos casos, que se reduce a que, en los centros privados, hay mayor número de sujetos que dicen sufrir la maledicencia de otros y también mayor número de sujetos que admiten hablar mal de sus compañeros, ponerles motes e ignorar a otros.

El hecho de vivir en una comunidad autónoma particular no influye en el grado de incidencia del maltrato entre iguales. Tampoco el hecho de que el hábitat en el que se ubique el centro de educación secundaria sea rural o urbano influye en la incidencia del maltrato.

Finalmente, es importante comparar estos datos con los que existen acerca de otros países europeos. Hay que señalar, antes de nada, que esta comparación resulta muy difícil por varias razones: en el cuestionario utilizado en el presente estudio se especifican mucho más los tipos de maltrato y, por tanto, las categorías no se corresponden directamente con las de otros cuestionarios; el período de tiempo por el que se pregunta puede variar según los estudios ${ }^{6}$. Hecha esta salvedad, se puede concluir que la incidencia del maltrato entre iguales en los centros de secundaria de nuestro país es relativamente baja. También se comprueba que el orden de incidencia de los distintos tipos de maltrato encontrado en los escolares españoles cuando hablan como víctimas coincide básicamente con el obtenido en otros estudios. Por lo que se refiere a las variables que permiten matizar estos datos de incidencia general, se observa que todos los resultados de la muestra española 
coinciden con los del resto de los estudios en lo que se refiere a género y edad. La mayor incidencia del maltrato se produce en el primer ciclo de la secundaria (entre 12 y 14 años) y va descendiendo paulatinamente hasta cuarto curso (16 años).

\section{3. ¿Dónde se produce el maltrato?}

Es importante saber dónde tienen lugar las agresiones entre compañeros para planificar la intervención en el centro. Cuando se pregunta a los estudiantes, discriminan claramente el lugar en el que se les maltrata, dependiendo del tipo de agresión sufrida. El aula se erige como el escenario más repetido de agresiones, si bien no todas se realizan en ella. De hecho, resulta el escenario privilegiado para los insultos y motes por un lado, y el acoso sexual por otro -con diferencias más que relevantes en su frecuencia relativa en el conjunto de la muestra estudiada-. Pero sobre todo tiene importancia para acciones contra las propiedades del alumnado, como romper o robar cosas.

Los aseos son el escenario en el que se esconden cosas. Varios tipos de maltrato como hablar mal de otros, la exclusión social, las amenazas sin armas, o pegar tienen escenarios más distribuidos dentro del centro -incluyendo el patio- y fuera de él. Las amenazas con armas, apenas existentes, tienen lugar fuera del centro con más frecuencia, y es en los aseos donde se realizan cuando ocurren en el centro escolar.

En la comparación de estos resultados con los datos suministrados por los docentes se observa que éstos no consideran tanto como sus estudiantes que el aula sea el escenario privilegiado del maltrato, en particular de agresiones graves (físicas directas, amenazas y chantajes y acoso sexual). Desconocen que los aseos son el lugar donde con mayor frecuencia esconden los alumnos las cosas de sus compañeros. La distinta visión que profesores y alumnos tienen del aula como escenario de abusos podría tener diversas explicaciones. Podría ser que los alumnos fueran menos fieles en su percepción de la incidencia, pero esta explicación es muy poco probable por lo que se sabe sobre abusos entre pares. Podría también deberse a una menor capacidad de los profesores para darse cuenta de lo que sucede en el aula, sobre todo en los momentos en que están ausentes, por ejemplo, en los cambios de clase.

\section{4. ¿Cómo se reacciona?}

Cuando una víctima es agredida por sus compañeros, tiende la mayoría de las veces a contárselo a sus amigos o amigas, teniendo esta 
comunicación una frecuencia mayor en el caso del acoso sexual y de las amenazas con armas. Esta importancia de los amigos como interlocutores de las agresiones que sufre la víctima permanece a través de los distintos tipos de maltrato, con porcentajes similares en todos ellos.

Alrededor de un $36 \%$ de los casos lo comunican a la familia, aunque esta frecuencia aumenta cuando el maltrato consiste en no dejar participar y en las amenazas para obligar a hacer algo y en las amenazas con armas. Al profesorado apenas se le cuenta lo ocurrido, aunque en las situaciones de amenazas aumenta el porcentaje de petición de ayuda, sobre todo cuando hay armas por medio o en las situaciones de acoso sexual. En cualquier caso, resultan cifras muy bajas considerando la gravedad de las situaciones.

En relación con la respuesta que las víctimas encuentran en quienes les rodean, con independencia del tipo de agresión sufrida, sólo cuentan con la ayuda de algún amigo o amiga. En cada uno de los tipos de agresión, más de un $60 \%$ de las víctimas mencionan esta ayuda, excepto en el caso de las amenazas con armas en que se recibe la ayuda de los amigos en algo menos de la mitad de los casos. No sólo los amigos intervienen para ayudar, aunque hay que señalar que el resto de la ayuda recibida supone sólo un tercio de la que proporcionan aquéllos. El papel de los padres y profesores aumenta en los casos de acoso sexual. Podría pensarse que la gravedad del maltrato hace que se incremente la ayuda por parte de todos los que puedan tener conocimiento del hecho. La gravedad también parece explicar el mayor porcentaje de quienes reconocen la ayuda de los profesores en tres tipos de maltrato: ser robado, pegado o amenazado sólo para meter miedo.

Con todo, no siempre los otros -ni siquiera siendo amigos- están ahí: aproximadamente en un $20 \%$ de los casos de exclusión, amenazas para obligar a hacer algo y amenazas con armas nadie ayuda a la víctima. Estos datos deben llevar a recomendar medidas de prevención que tengan en cuenta las relaciones interpersonales, entre ellas, el fomento de actitudes prosociales no interesadas, es decir, no prestadas exclusivamente a los amigos.

Es también muy interesante analizar lo que los alumnos dicen acerca de sus propias reacciones cuando contemplan estos conflictos en quienes les rodean. De acuerdo con los testigos del maltrato, un $50 \%$ interviene para cortarlo cuando es un amigo, porcentaje que se reduce cuando no tienen un lazo de amistad con la víctima. En menos del $10 \%$ de los casos se informa a algún adulto. Pero además no todos aquéllos que presencian el maltrato a un compañero o compañera responden con un intento de que éste no vaya a más. Así, un 18 '5\% no hace nada, aunque buena parte de ellos piensa que debería hacerse algo (14\% de 
la muestra total). Todavía más: un porcentaje pequeño de testigos -1 '5\% de los casos, que tratándose del maltrato nunca se puede considerar irrelevante- confiesan que lo que hacen al ver la agresión es meterse con la víctima, convirtiéndose en cómplices deliberados del autor primario del maltrato.

Cuando se pregunta a los estudiantes sobre la reacción de los docentes, la respuesta más frecuente es que algunos profesores intervienen para cortarlo, aunque menos de un tercio de la muestra total opta por ella. En un cuarto de la muestra no se sabe lo que hacen y en otro cuarto se afirma que no hacen nada porque no se enteran; una quinta parte de los encuestados afirma que los profesores castigan a los que agreden. Parece pues que el profesorado tiene un papel menos relevante que los compañeros a la hora de intervenir para que no siga la escalada cuando se da una agresión. Lo que, unido al dato de que, según los estudiantes, ignora la mayor parte de las veces que se estén produciendo estos conflictos, confirma los resultados obtenidos en otros países acerca de este fenómeno como algo soterrado que permanece en el mundo no adulto y que forma parte del currículum oculto de las escuelas.

\subsection{Conflictos entre profesores y alumnos.}

Aunque, como se ha señalado más arriba, el objetivo del estudio era fundamentalmente conocer la incidencia del maltrato entre compañeros, en los cuestionarios se preguntó a profesores y alumnos por otro tipo de conflictos en los centros, en concreto, por las agresiones que se producían entre unos y otros. A continuación se resumen los principales resultados.

Los resultados relativos a agresiones de los alumnos hacia los docentes muestran que la forma más habitual en la que este tipo de conflicto se produce es el insulto. "Sembrar rumores dañinos" y "destrozar enseres" tienen también frecuencias muy altas. El robo es igualmente un tipo de agresión que en opinión de los profesores sucede con una cierta probabilidad. Incluso la intimidación con amenazas tiene cierta presencia. Pero lo más preocupante es el hecho de que también tienen lugar en los centros agresiones físicas directas, aunque la frecuencia sea escasa.

La otra cara de la moneda viene dibujada por los datos sobre agresiones de profesores a alumnos. En este caso la forma de agresión más habitual es ridiculizar al estudiante. También es bastante frecuente "insultar" y "tener manía". La intimidación con amenazas alcanza un $24 \%$. Las agresiones menos frecuentes son las físicas y las que consisten en sembrar rumores dañinos, pero desgraciadamente no puede 
decirse que no se produzcan (un 6'3\% del profesorado señala en ambos casos que "a veces ocurre"). Allí donde la comparación es posible en relación con las agresiones de alumnos a profesores, se observa una semejanza llamativa en algunas categorías. Semejanza que resulta preocupante dada la clara asimetría de la relación profesor-alumno y que, en ambos casos, son datos que los propios docentes suministran. También hay que tener en cuenta, no obstante, al interpretar este dato que la muestra es pequeña y que el jefe de estudios que contesta al cuestionario tiene como referencia al conjunto de los docentes de su centro. Cuando contesta que estas agresiones tienen lugar, pudiera estar pensando en profesores o profesoras aislados que no fueran representativos del claustro. En cualquier caso, lo que estos datos ponen de manifiesto es que el problema remite al clima de convivencia general de los centros y no se limita a un único tipo de agresiones.

\subsection{Las ideas de los docentes acerca de la importancia del mal-} trato entre alumnos y de cómo afrontarlo.

Ante la pregunta de si creen que los conflictos han aumentado en su centro en los tres últimos años, un $45 \%$ del profesorado contesta que "ligeramente" y un $32 \%$ considera que el aumento ha sido drástico. Sin embargo, cuando tienen que graduar la importancia que los conflictos y agresiones entre alumnos tienen en el funcionamiento de su centro, en comparación con otra serie de problemas muy habituales en las instituciones docentes, no lo consideran como uno de los principales, dando prioridad a las dificultades de aprendizaje de los estudiantes, la falta de participación de las familias y la falta de recursos humanos y materiales.

Cuándo se les pregunta a los docentes cuáles creen que son las medidas que se deberían tomar para prevenir situaciones de maltrato entre iguales, los recursos en que más confían los docentes parecen ser la tutoría y la iniciativa, relacionada con ella, de trabajar y debatir en clase las normas del Reglamento de Régimen Interno. Este dato es muy positivo desde un enfoque preventivo y no sancionador de la intervención. Hay que hacer notar, no obstante, que los profesores de secundaria tienen todavía una actitud ambivalente hacia la tutoría, que en ocasiones les lleva a un cierto rechazo de esta tarea, aunque afortunadamente la tendencia es a ir valorando cada vez más este elemento educativo básico.

Las otras dos medidas que consiguen mayor consenso en el profesorado son también muy adecuadas dentro de un enfoque preventivo. La importancia otorgada a "sensibilizar a los profesores acerca de las características personales de los alumnos" parece ilustrar la toma de 
conciencia por parte del profesorado de secundaria de la necesidad de mejorar su preparación en este campo y también la aceptación de que educar exige tener en cuenta el conjunto del desarrollo del alumno y no sólo los aspectos relacionados más directamente con cada materia específica del currículum. Los profesores también son partidarios de favorecer una metodología más participativa, dirigida a motivar a los alumnos y a mejorar la convivencia en el aula. Lo que llama, sin embargo, la atención a este respecto es la desconfianza que los profesores parecen tener en que la formación del profesorado sea una manera eficaz de prepararse en estos dos ámbitos señalados.

Las opiniones recogidas acerca de cómo prevenir y enfrentar los problemas de violencia entre iguales en los centros nos dan algunas pistas que ayudan a planificar las posibles medidas que podrían tomarse desde la Administración educativa y desde las propias comunidades escolares. En lo que sigue se resumen las principales ideas a este respecto.

\section{CÓMO MEJORAR LA CONVIVENCIA EN LOS CENTROS ESCOLARES.}

Ya se ha señalado más arriba que la mejor manera de prevenir los conflictos es establecer un buen clima de convivencia en los colegios e institutos. Los enfoques meramente sancionadores se han venido mostrando totalmente insuficientes e inadecuados para afrontar los conflictos escolares (Fernández, 1998, Smith y otros, 1999). Los modelos preventivos, por el contrario, se caracterizan por actuar en el conjunto del centro y no en elementos aislados, y por centrarse en las medidas relacionadas con la organización, la cultura y el currículum de la institución (Torrego, 2000).

La LOGSE y su desarrollo normativo adolecieron de una reflexión a fondo sobre los cambios en la estructura de los centros que eran indispensables para llevar a la práctica las innovaciones que se proponían (Marchesi y Martín, 1998). La LOPEG enfrentó el problema en alguno de sus aspectos, pero se ha mostrado insuficiente. El enfoque del Real Decreto de Derechos y Deberes de los Alumnos resulta un marco con una tendencia burocrática y fundamentalmente sancionadora que lleva a olvidar en muchos casos la función educativa de cualquier intervención en un centro escolar y a actuar solamente cuando los problemas se han planteado ya. Es preciso pues plantear el problema en una perspectiva más amplia en la que se asuma plenamente que el clima de convivencia de las instituciones docentes es un recurso de aprendizaje básico y debe actuarse sobre él para mejorar la enseñanza 
de todos los alumnos y alumnas.

Un primer paso que se señala en todos los documentos teóricos escritos en este campo y en los programas que ya vienen poniéndose en marcha en los últimos años (Defensor del Pueblo, 2000; Ortega y otros, 1998; Smith y otros, 1999) es la necesidad de que se tome conciencia del problema. Gran parte de los conflictos permanecen en el terreno del currículum oculto de los centros y se observan claras resistencias a enfrentarse directamente con el problema así como un desconocimiento bastante generalizado del mismo entre docentes y familias. En este momento contamos ya con un número importante de estudios que se sirven de variados instrumentos (cuestionarios, entrevistas semiestructuradas, análisis de narraciones) y permiten hacerse una imagen global no sólo de la situación en la que se encuentran las relaciones interpersonales en el centro, sino también de cómo se representan estos problemas los propios estudiantes. Esta información debe ser el punto de partida para reflexionar acerca de las explicaciones que profesores, familias y estudiantes dan al problema. No es un proceso sencillo, ya que remueve creencias muchas veces implícitas que no resulta fácil reconocer y menos cambiar, pero es imprescindible partir de una valoración conjunta sobre la situación en la que se encuentra el clima de convivencia en el centro.

Los centros deben repensar las decisiones del Proyecto Educativo que afectan a la convivencia. Las relaciones con las familias, el aprovechamiento de los recursos que el entorno social les ofrece para llevar a cabo actividades con los alumnos positivas para su desarrollo social (extraescolares, programas de juventud, colaboración con ONGs, etc.), los valores que van a presidir la actuación educativa, los canales que van a permitir una auténtica participación de todos los sectores de la comunidad escolar, son algunos de los elementos fundamentales, pero quizás el punto central se encuentra en la elaboración del Reglamento de Régimen Interno. Es imprescindible hacer de este documento un instrumento real de mejora de la convivencia. En él deben establecerse los cauces para favorecer las relaciones interpersonales y, en caso de conflicto, resolverlo de forma que todos aprendamos lo más posible en el proceso (Casamayor, 1998).

Es importante también que los contenidos del currículum sean consecuentes con este enfoque. La educación en valores, los temas transversales, el trabajo sobre capacidades afectivas y sociales, y no meramente sobre las intelectuales, son requisitos para desarrollar en los alumnos y alumnas capacidades prosociales sobre las que construir relaciones interpersonales positivas. En algunos programas se propone llevar a cabo cursos específicos de habilidades sociales. Sin mostrar un rechazo frontal a este tipo de medidas, consideramos que resulta mu- 
cho más adecuado trabajar estas destrezas con todos los alumnos y desde el conjunto de las asignaturas. Depositar esta responsabilidad exclusivamente en un docente o experto que imparte el curso al margen del resto del equipo de profesores limita drásticamente la eficacia de la intervención.

El plan de acción tutorial constituye otra de las piezas clave del problema. Es fundamental trabajar las cuatro dimensiones que la tutoría supone para prevenir los conflictos: el seguimiento individual y personalizado del alumno o alumna, el trabajo con el grupo-clase, la comunicación con la familia y la coordinación del equipo educativo. La tutoría es un momento especialmente adecuado para reflexionar sobre las relaciones interpersonales y los profesores así lo reconocen, como hemos visto en el apartado anterior, pero la acción tutorial es mucho más que la hora de tutoría y debe planificarse de forma global para atender al desarrollo integral de cada uno de los estudiantes, lo que supone ocuparse de su progreso social y moral. En relación con esto, una propuesta llena de interés es el conjunto de actividades diseñado por Sastre y Moreno (2002) para trabajar en el aula. Todas las actividades están basadas en conflictos inspirados en el día a día como punto de partida significativo para la reflexión, la aportación de estrategias de resolución positivas y, en definitiva, el aprendizaje emocional como objetivo educativo.

La mayor parte de estas medidas no resultan novedosas en teoría, ya que están previstas en las normas que rigen el funcionamiento de los centros. Pero no han calado realmente en la vida escolar por falta de los apoyos adecuados y de las condiciones de organización de los centros que las hagan posibles. Una función directiva que no termina de asumir su tarea de liderazgo pedagógico, unos horarios escolares que impiden momentos educativos que no sean los meramente lectivos, un exceso de profesores impartiendo clase a un mismo grupo, una insuficiente preparación del profesorado para desarrollar la función tutorial, una escasa participación de los alumnos y de sus familias en las decisiones del centro -no siempre por falta de interés-, son algunos de los factores que pueden explicar el fracaso de estas propuestas.

Junto con las actuaciones anteriores se están poniendo en marcha también otras más específicas dirigidas a la prevención y resolución de conflictos, entre las que nos limitaremos a citar las dos que nos parecen más interesantes: los programas de mediación y la ayuda entre iguales. La mediación, que está mostrando un camino fructífero en otros ámbitos sociales, se va abriendo camino también en el contexto escolar (Uranga, 1998; Fernández y Funes, 2001). En algunos casos, todavía muy reducidos, se están llevando a cabo mediaciones formales utilizando como mediadores a docentes, estudiantes o familiares que 
han recibido formación específica para llevar a cabo esta tarea. Algunas administraciones están preparando a determinados profesionales (inspectores, asesores psicopedagógicos...) para actuar como mediadores en determinados tipos de conflictos en los centros. En otros casos la actuación responde más a una mediación informal que permite una respuesta más rápida y más "natural" para lo que es la dinámica escolar. Todavía es pronto para hacer una valoración rigurosa de este enfoque de intervención, pero lo que hasta ahora se ha analizado arroja una valoración positiva.

Por lo que se refiere a los programas de ayuda entre iguales (Cowie, 1995; Cowie y Sharp, 1996; Cowie y Wallace, 2000; Fernández y Funes, 2001), en los que los propios alumnos sirven de recurso para resolver conflictos o apoyar a otros compañeros que lo necesiten por cualquier motivo, resulta una perspectiva muy interesante por diferentes razones. En primer lugar son coherentes con uno de los datos que obtenía con mayor claridad el estudio del Defensor del Pueblo: Ios alumnos recurren sobre todo a sus compañeros cuando son víctimas de alguna agresión. Por otra parte, los iguales son un referente básico durante el desarrollo, sobre todo en la adolescencia (Moreno y del Barrio, 1999). Por último, los estudios que se han llevado a cabo acerca del funcionamiento de estos programas (Naylor y Cowie, 1999; Salmivalli, 1999) ponen de manifiesto que consiguen hacer progresar las habilidades sociales de los alumnos que actúan como ayudantes o apoyo del resto de grupo. Es importante que estos programas permitan asumir este papel a la mayor parte de los alumnos para que todos puedan beneficiarse de estos aprendizajes y evitar con ello además que puedan formarse "élites" que sean vividas por el resto como grupos de privilegiados en lugar de recursos a su servicio.

Las medidas señaladas hasta aquí no agotan en absoluto las actuaciones que pueden llevarse a cabo en los centros, pero permiten mostrar, desde nuestro punto de vista, la importancia de planificar la mejora de la convivencia en los centros y ponen de manifiesto también que existe ya un importante bagaje de conocimiento sobre el tema que puede permitir a los centros acometer la tarea con perspectivas de éxito. 


\section{BIBLIOGRAFÍA.}

BARRIO, C. del; MARTÍN, E.; MONTERO, N.; FERNÁNDEZ, I.; GUTIERREZ, H. y OCHAÍTA(2002): La realidad del maltrato entre iguales en la educación secundaria. Infancia y Aprendizaje, en prensa.

CASAMAYOR, G. (coord. ) (1998): Cómo dar respuesta a los conflictos. Barcelona: Graó.

COWIE, H. y SHARP, S. (1996): Peer Counselling in Schools. Londres: David Fulton Publishers.

COWIE, H. y WALLACE, P. (2000): Peer Support in action. Londres: Sage Publications.

DEFENSOR DEL PUEBLO (2000): Informe sobre violencia escolar: el maltrato entre iguales en la educación secundaria obligatoria. Elaborado por C. del Barrio, E. Martín, I. Montero, L. Hierro, I. Fernández y H. Gutiérrez, por encargo del Comité Español del UNICEF. Madrid: Publicaciones de la Oficina del Defensor del Pueblo. Versión electrónica: www.defensordelpueblo.es/informes/espec99/maininfoal

FERNÁNDEZ, I. (1998): Prevención de la violencia y resolución de conflictos. El clima escolar como factor de calidad. Madrid: Narcea.

FERNANDEZ, I. y FUNES, S. (2001): Sistemas de ayuda entre iguales y de resolución de conflictos para la mejora de la convivencia. Los problemas de convivencia escolar: un enfoque práctico. Madrid: CCOO.

MARCHESI, A. y MARTÍN, E. (1998): Calidad de la enseñanza en tiempos de cambio. Madrid: Alianza.

MORENO, A. y del BARRIO, C. (2000): La experiencia adolescente. Buenos Aires: Aique.

NAYLOR, P. y COWIE, H. (1999): The effectiveness of peer support systems in challenging school bullying: the perspectives and experiences of teachers and pupils. Journal of Adolescence, 22, 467-479.

OLWEUS, D. (1993): Bullying at school: What we know and what we can do. Oxford.: Blackwells. Trad. Cast.: Conductas de acoso y amenaza entre escolares. Madrid: Morata, 1998.

ORTEGA, R. et al. (1998): La convivencia escolar: qué es y cómo abordarla. Sevilla: Consejería de Educación y Ciencia, Junta de Andalucía.

SALMIVALLI, C. (1999): Participant role approach to school bullying: implications for interventions. Journal of Adolescence, 22, 453-459.

SASTRE, G. y MORENO, M. (2002): Resolución de conflictos y aprendizaje emocional. Una perspectiva de género. Barcelona: Gedisa.

SMITH, P.K.; MORITA, Y.; JUNGER-TAS, J.; OLWEUS, D.; CATALANO, R. y SLEE, P. (eds) (1999): The Nature of School Bullying. A cross-national perspective. Londres: Routledge. 
TORREGO, J.C. (2000): Mediación de conflictos en instituciones educativas. Madrid: Narcea.

URANGA, M. (1998): Mediación, negociación y habilidades para el conflicto en el marco escolar. En G. Casamayor (coord.) (1998).

Van der MEULEN, K.; SORIANO, L; GRANIZO, L.; del BARRIO, C.; KORN, S. y SCHÄFER, M. (2002): Los efectos de la victimización entre escolares: un enfoque retrospectivo. Infancia y Aprendizaje, en prensa. 